\title{
Endogenous Risks and Learning in Climate Change Decision Analysis
}

Brian C. O’Neill (oneill@iiasa.ac.at)

Yurii Ermoliev (ermoliev@iiasa.ac.at)

Tatiana Ermolieva (ermol@iiasa.ac.at)

\section{Approved by}

Leen Hordijk

Director

October 5, 2005 Institute, its National Member Organizations, or other organizations supporting the work. 


\section{Contents}

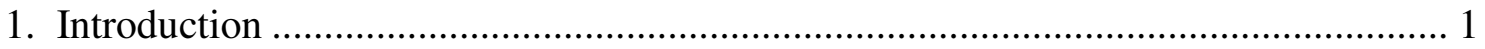

2. Endogenous Climate Change Risk .......................................................................... 3

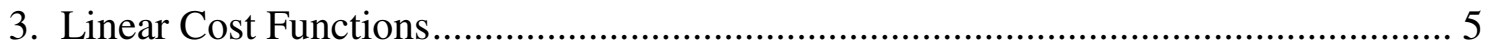

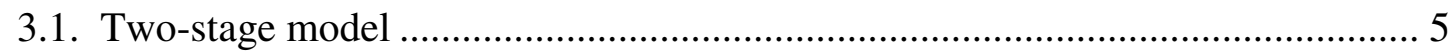

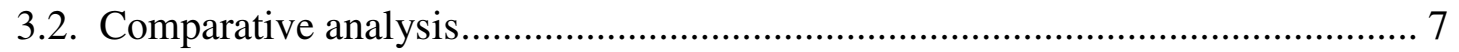

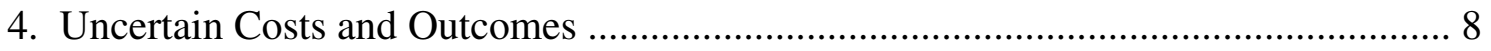

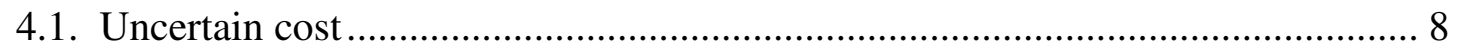

4.2. Uncertain durations of stages: Limited adaptive capacity ................................... 9

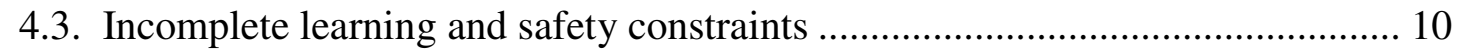

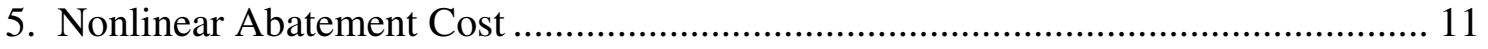

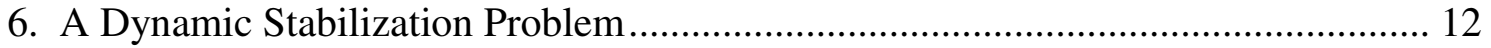

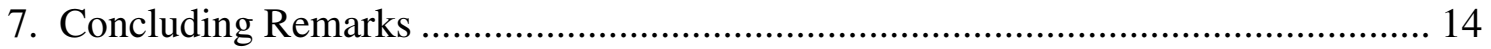

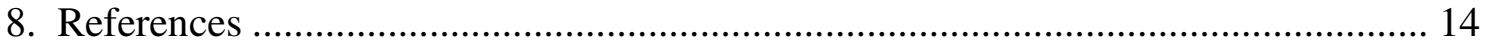




\section{Abstract}

We analyze the effects of risks and learning on climate change decisions. A two-stage, dynamic, climate change stabilization problem is formulated. The explicit incorporation of ex-post learning induces risk aversion among ex-ante decisions, which is characterized in linear models by VaR- and CVaR-type risk measures. Combined with explicit introduction of "safety" constraints, it creates a "hit-or-miss" type decisionmaking situation and shows that, even in linear models, learning may lead to either lessor more restrictive ex-ante emission reductions. We analyze stylized elements of the model in order to identify the key factors driving outcomes, in particular, the critical role of quantiles of probability distributions characterizing key uncertainties. 


\section{Acknowledgments}

This paper has been submitted for publication in an edited volume of peer-reviewed papers based on presentations at the IFIP/IIASA/GAMM workshop on "Coping with uncertainty", December 13-16, 2004, IIASA. 


\title{
Endogenous Risks and Learning in Climate Change Decision Analysis
}

\author{
Brian C. O'Neill \\ Yurii Ermoliev \\ Tatiana Ermolieva
}

\section{Introduction}

Discussions of climate change policies are often framed as a choice between acting now or waiting until we know more about the problem (Manne and Richels, 1992; Webster 2002; Wright and Erickson, 2003). The climate change policy dilemma poses a "hit-ormiss" type of decision making situation. Emissions of greenhouse gases (GHG) are associated with the production and consumption of goods and services, and the atmospheric concentration (stock) of these long-lived pollutants may cause irreversible damages. On one hand, postponing the reduction of GHG emissions may lead to potentially irreversible climate-related impacts, for example, reorganizations of largescale ocean circulation patterns or increased frequency of extreme weather-related events. On the other hand, if the problem turns out to be less severe than expected, the delay will avoid irreversible investments in capital for emissions abatement.

These discussions are often supported by a traditional expected utility maximization model, suggesting either risk-averse ex ante (anticipative) decisions or risk-prone ex post (adaptive) decisions that are made only after receiving full information. If uncertainty about climate change is resolved over time, the robust strategy would definitely be to make only partial ex ante commitments and to keep options open until new information is revealed. This approach requires so-called twostage recourse stochastic optimization (STO) models (Dantzig and Madansky, 1961; Ermoliev and Wets, 1988; Kall and Wallace, 1994; Yastremskij 1983) incorporating both ex ante and ex post decisions within a single model. Accordingly, the climate change stabilization problem (Section 2) can be formulated as the choice of an ex ante risk reduction (stage 1) strategy over a random time horizon taking into account that it may need to be adjusted after new information is revealed (stage 2). This decisionmaking framework implies, in particular, that the capacity for adaptive decision making in stage 2 has to be created ex ante.

In economics literature, the importance of learning was first discussed in connection with irreversible investments in 1974 in Arrow and Fisher (1974) and Henry (1974) without an overall two-stage model being formulated. Arrow and Fisher (1974), Henry (1974), and Chichilnisky and Heal (1993) have concluded that when future damages are uncertain and irreversible, the ability to learn should lead to more active $e x$ ante emission reductions. On the other hand, irreversibility of capital may lock an 
economy into a wasteful use of resources. Viscusi and Zeckhauser (1976), Dixit and Pindyck (1994), Ulph and Ulph (1997), and Pindyck (1999) showed that the ability to learn in this case should lead to less active ex ante emission reduction. These competing effects imply that the net effect of learning on ex ante decisions is an empirical question. Nordhaus (1994) and Kolstad (1996) examined the effects of learning by using empirically-calibrated integrated assessment models. They concluded that, in fact, learning has insignificant effects on ex ante abatement policies because the damage losses are not severe enough. A reason for this is that in most integrated climate and economics models, climate changes are considered as if they occur continuously and as if they can eventually be reversed through ex post adjustments (Wright and Erickson, 2003). These models also use average damages (i.e., they cannot properly capture the effects of abrupt climate change and catastrophic risks (Ermoliev et al, 2000; O'Neill and Oppenheimer, 2002; Wright and Erickson, 2003)). A paper by Fisher and Narain (2003) analyzes a two-period model with risk characterized by a parameter introducing high or low climate change damages. Because overall impacts are evaluated by using expected values, the effects of capital irreversibility dominate catastrophic damages in a similar way to other models. Epstein (1980) demonstrated that the effects of learning on ex ante decisions depend in general on the convexity or concavity of marginal costs, which are very restrictive for climate change policy analysis (Ulph and Ulph, 1997).

In this paper we take a different approach. Instead of using expected damages we explicitly introduce safety constraints by formulating the climate change problem within the framework of stabilization. We develop a two-stage dynamic STO model and deliberately analyze only stylized linear versions of this model. We show that the combination of safety constraints and perspectives of learning induces potentially strong risk aversion among ex ante decisions that is characterized by quantile-based VaR and $\mathrm{CVaR}$ risk measures. As a result we show that, even with a linear net cost function, learning may lead to either less or more restrictive emission reductions, depending on mitigation costs and probability distributions describing key uncertainties.

Section 2 characterizes climate change risk by the probability of total atmospheric $\mathrm{CO}_{2}$ concentrations exceeding a vital random threshold associated with potential ranges of global temperature. It outlines a two-stage dynamic climate change stabilization STO model with random durations of stages. In general, this model can be solved only numerically and therefore the key factors driving results are difficult to identify. For these reasons, we analyze only stylized aspects of the model; these provide a clearer picture of the various driving forces and show why the ability to learn in the future can lead to either less-restrictive or more-restrictive ex ante abatement policies today.

Section 3 uses a very simple linear two-stage STO model to illustrate that the results from empirical models can be rather contradictory, because optimal solutions depend on complex nonsmooth interactions among ex ante and ex post decisions, costs, and probability distributions. In particular, they induce potentially strong risk aversion characterized by risk measures that are used for regulating the safety of nuclear plants and insolvency of insurance companies, but also in financial applications, extremal value theory (Embrechts et al, 2000), and catastrophic risk management (Ermoliev et al, 2000). 
Section 4 analyzes the effects of uncertain costs and random duration of stages. It emphasizes the importance of quantiles of probability distributions characterizing key uncertainties and shows that without aggressive ex ante commitments, a misperception of limited adaptive second-stage capacity may provoke a catastrophe (i.e., exceedance of the safety constraint).

Sections 4 and 5 discuss the controversial effects of incomplete learning and nonlinear costs, which more strongly and even unconditionally require coexistence of $e x$ ante anticipative and ex post adaptive risk-management decisions.

A more realistic but still linear dynamic two-stage climate change stabilization STO model will be analyzed in Section 6. Similar to Section 3, the explicit incorporation of ex ante and ex post decisions induces risk aversions characterized by a dynamic version of a CVaR type risk measure. This may create a wrong impression of truly risk-based policy analysis and, without explicit introduction of adaptive capacity and additional safety constraints, may provoke a catastrophe. In conclusion, Section 7 emphasizes the importance of the proper treatment of uncertainty, risks, and robust decisions, as well as the development of adequate computational approaches.

\section{Endogenous Climate Change Risk}

Climate change integrated assessment models (see, e.g., (Nordhaus, 1994)) incorporate economic and geophysical processes that link economic growth models with the accumulation of GHG emissions in the atmosphere. The accumulation of $\mathrm{CO}_{2}$ emissions is the main driving force behind global climate change. The process involves complex interactions between the atmosphere, the upper oceans and the biosphere, and the deep oceans. Current integrated assessment models use different carbon cycle parameterizations for computing changes in atmospheric concentrations $\mathrm{M}(\mathrm{t})$ resulting from $\mathrm{CO}_{2}$ emissions $e(t)$ (Schultz and Kasting, 1997). In general, these models are of the form

$$
M(t+1)=f[M(t), e(t), \beta], t=0,1,2, \ldots .
$$

where $\beta$ is a vector of model parameters. Values $M(t)$ are used in integrated assessment models to compute the increase in the global average temperature as a smooth function of $M(t)$, and damages are typically computed in the form of smooth functions of this temperature increase. The resulting smooth dependence of damage functions on global average temperature does not permit the proper modeling of abrupt climate changes. A serious underestimation of climate change impacts may also result from the use of average global temperature. It is possible that changes in average global temperature would be associated with an increase in the frequency of extreme weatherrelated events or could trigger singular events with widespread consequences (e.g., the disintegration of the West Antarctic Ice Sheet (O'Neill and Oppenheimer, 2002)). Therefore, proper modeling of risks is crucial for evaluating climate-change policies.

Emissions $e(t)$ in (1) depend on a vast variety of uncertainties, denoted by $\omega$, and policy variables, denoted by $x$. In this paper, we assume that emissions $e(t, \omega)$, $t=0,1, \ldots$, are characterized as random variables defined in a probability space $\Omega$ of scenarios $\omega, \omega \in \Omega$, with a probability measure $P(d \omega)$. Thus, for $\Omega=\{1, \ldots, N\}$, 
$P(d \omega)=p(s):=\mathrm{P}[\omega=s], \quad \sum_{s=1}^{N} p(s)=1 . \quad$ Frequently we do not indicate the dependence of random variables on $\omega$ if this is clear from the context.

We introduce risk by imposing a safety constraint in the form of an atmospheric stabilization target, beyond which it is assumed that the risks of high-impact outcomes substantially increase. Let us denote by $L(\omega)$ the uncertain target level of $\mathrm{CO}_{2}$ concentration in the atmosphere. The main problem can be formulated as the choice of a cost-efficient emission-reduction path that satisfies probabilistic safety constraints on vital but uncertain levels of concentrations

$$
\mathrm{P}[M(t, x, \omega) \leq L(\omega), t=\overline{1, T}] \geq 1-\gamma,
$$

where $\gamma$ is a risk factor, $0 \leq \gamma<1, T$ is a time horizon that may also be uncertain. In the insurance industry, constraints of type (2) regulate risk reserves to prevent insolvency. The typical approach to choosing $\gamma$ in this industry is not based primarily on evaluating potential damages, but rather on limiting the chance that the insolvency may occur to, say, once in 800 years, $\gamma=1 / 800$. Similarly, the major failure of a nuclear plant is allowed once in $10^{7}$ years, $\gamma=10^{-7}$. Note that these are expected time horizons, and therefore there is the possibility that events may occur at any time.

The concentration-stabilization problem can be formulated in a similar way to the catastrophic risk management problem discussed in (Ermoliev et al, 2000). At stage 1 , the emission-reduction path is defined by ex ante decisions $x(t), t=1,2, \ldots$ until a random time moment $\tau$ when new information about uncertain variables is revealed. The new information may also include a new critical time horizon $T(\omega)$ for stage 2 ex post emission reductions $x(t, \omega), t=\tau+1, \ldots, T(\omega)$. The problem is to minimize total emission reduction cost.

The resulting model can only be solved numerically. Here, instead of using numerical simulations, we take a different approach. In the following sections we formulate various stylized elements of the model and evaluate them analytically. This allows us to keep the discussion on a simple level, which provides a clear picture of the potential results.

Remark 1: The abrupt climate change in (2) is modeled by random $L(\omega)$, which is revealed as a "shock" at random moment $\tau(\omega)$. Despite a smooth and even linear dependence of function $M(t, x, \omega)$ on $x$, the left-hand side of (2) is, in general, a nonsmooth and often even a discontinuous risk function (Ermoliev and Norkin, 1997; Ermoliev and Wets, 1998; Marti, 2005). Endogenous catastrophic collapse is modeled as a violation of constraint (2). In general, the learning may not reveal full information but perhaps only shift ranges of probability distributions. The learning may also not occur at $\tau(\omega) \leq T$, or it may occur very close to $T(\omega)$. Since the inertia of the system may not allow constraints (2) to be fulfilled quickly, the probability of a catastrophe conditional on revealed information may drop rapidly below the vital level $\gamma$ (i.e., constraint (2) emphasizes the importance of proper ex ante actions). 


\section{Linear Cost Functions}

The following two-stage model is relatively easy to analyze. It provides suggestive results and serves as a building block of more general two-stage dynamic models (Section 7). Even in its simplest form, it already shows that results from purely empirical models can be contradictory regarding the effects of learning on ex ante decisions, since optimal solutions depend on complex nonlinear interactions among decisions, costs, and probability distributions that characterize uncertainties.

\subsection{Two-stage model}

A stylized climate change stabilization problem can be formulated as follows: assume that there are only two time intervals or periods $t=1,2$. Define by $x_{t}, x_{t} \geq 0, t=1,2$, a feasible level of emission reduction that can be chosen in period $t ; C_{t}, C_{t}>0$ is the known expected abatement cost per unit of emission reduction in period $t ; \theta(\omega)$ is the uncertain target value of cumulative emission reductions for two periods. In this problem formulation, $\theta(\omega)$ serves as the safety constraint, $x_{1}+x_{2} \geq \theta(\omega)$. The constraint on minimum emissions reduction can be thought of as a concise way to represent several factors (and their uncertainties) that come into play in meeting a target based on environmental outcomes such as atmospheric concentrations, global average temperature levels, or particular impacts. For example, emissions reductions required to meet a target will depend on the target itself (i.e., whether a concentration or temperature target is high or low), on reference emissions e(t) (because the absolute size of required emissions reductions will depend on the magnitude of uncontrolled emissions in the reference case), and on the system mapping emissions to environmental outcomes (e.g., parameters of the carbon cycle or climate system). Uncertainty in $\theta(\omega)$ can be thought of as reflecting uncertainty in one or more of these different factors.

Assume uncertainty in $\theta(\omega)$ is resolved between periods 1 and 2. The ex ante decision $x_{1}$ is made before the uncertainty in $\theta$ is resolved, whereas the ex post decision $x_{2}$ is based on known $\theta$ (i.e., $x_{2}$ is a function of $\left.\theta, x_{2}(\theta)\right)$. Assume that the ability to fulfill risk constraint $x_{1}+x_{2} \geq \theta(\omega)$ in period 2 is unbounded. The impacts of this rather unrealistic assumption (which is often a standard assumption of existing integrated assessment model) are analyzed in Section 4. The problem is formulated as the minimization of total linear costs

$$
C_{1} x_{1}+C_{2} \operatorname{Ex}_{2}(\theta)
$$

subject to safety constraints

$$
x_{1}+x_{2}(\theta) \geq \theta, \text { for all } \theta \text {. }
$$

Clearly, if the ex ante decision $x_{1}$ is irreversible, then the optimal period 2 decision is $x_{2}^{*}(\theta)=\max \left\{0, \theta-x_{1}\right\}$, that is, it nonsmoothly depends on period 1 decision $x_{1}$ (path dependence) and $\theta$, providing potentially strong cross-period random 
interactions among decisions. Optimal period-1 decision $x_{1}^{*}$ solves the stochastic minimax problem: minimize

$$
F(x)=C_{1} x+C_{2} E \max \{0, \theta-x\}, x \geq 0 .
$$

Remark 2: Although the initial model (3)-(4) is linear in $\left(x_{1}, x_{2}\right)$, the introduction of ex post decision $x_{2}$ induces risk aversion among ex ante decisions that is defined by implicit nonsmooth (in general) function (5). The following Proposition summarizes some important facts about stochastic minimax problem (5). It shows that the induced risk attitudes are characterized by $\mathrm{VaR}$ (critical quantile) and CVaR risk measures (Rockafellar and Uryasev, 2000). In extremal value theory (Embrechts et al, 2000), CVaR is also known as Mean Shortfall and Mean Excess Loss.

\section{Proposition:}

(i) $F(x)$ is a convex function. If $H(z)=\mathrm{P}[\theta \leq z]$ is a continuously differentiable function, then $F(x)$ is a strictly convex continuously differentiable function.

(ii) If $C_{1}>C_{2}$, then $x_{1}^{*}=0$ and $x_{2}^{*}(\theta)=\theta$. If $C_{1}<C_{2}$, then the necessary and sufficient condition for optimal $x^{*}$ reads: $x^{*}$ is the quantile satisfying equation

$$
P[\theta \geq x]=C_{1} / C_{2} .
$$

(iii) The optimal value $F\left(x^{*}\right)$ has two important representations:

$$
F\left(x^{*}\right)=C_{1} \bar{\theta}+C_{1} E\left[x^{*}-\theta \mid \theta \leq x^{*}\right]=C_{2} E \theta I\left(\theta \geq x^{*}\right),
$$

where $E[\cdot \mid \cdot]$ denotes the conditional expectation, the indicator function $I(\theta>x)=1$ if $\theta \geq x$ and $I(\theta \geq x)=0$ otherwise.

Let us outline the proof.

(i) The convexity of $F(x)$ follows from the convexity of function $\max \{0, \theta-x\}$ which is preserved under expectation operation. The strict convexity of $F(x)$ follows from the continuous differentiability of $F(x)$.

(ii) The minimization of $F(x)$ is a specific case of so-called stochastic minimax problems [10]. From the general results it follows that $F^{\prime}(x)=C_{1}-C_{2} P[\theta \geq x]$. From $C_{1}<C_{2}$, it follows that $F^{\prime}(0)<0$, i.e., $x^{*}>0$ (assuming $x^{*}=0$ we can derive a contradiction with assumption $C_{1}<C_{2}$ for small $x$ ). As $F(x)$ is a strictly convex function, it follows that (6) is indeed a necessary and sufficient optimality condition.

(iii) The first representation in (7) follows from (6) and the following rearrangements:

$$
\begin{aligned}
F\left(x^{*}\right) & =C_{1} x^{*}+C_{2} E \max \left\{0, \theta-x^{*}\right\}=C_{1} x^{*}+C_{2} E\left[\theta-x^{*} \mid \theta>x^{*}\right] P\left[\theta>x^{*}\right]= \\
& =C_{1} x^{*}+C_{1}\left(E\left[\theta-x^{*}\right]-E\left[\theta-x^{*} \mid \theta \leq x^{*}\right]\right)=C_{1} \bar{\theta}+C_{1}\left(E\left[x^{*}-\theta \mid \theta \leq x^{*}\right]\right) .
\end{aligned}
$$


The second representation in (7) follows from (6) and $E \max \left\{0, \theta-x^{*}\right\}=E \theta I\left(\theta \geq x^{*}\right)-x^{*} P\left(\theta \geq x^{*}\right)$.

Remark 3: The critical quantile in (6) defines the $\mathrm{VaR}$ risk measure, i.e., it indicates the magnitude of emission reduction in stage 1 that, with probability $1-C_{1} / C_{2}$, will be sufficient to meet the safety constraint with no additional reduction required in stage 2. The second equation in (7) defines the CVaR risk measure; i.e., the expected value of abatement costs that will be necessary in stage 2 if emissions reductions in stage 1 are not sufficient to meet the safety constraint. For some distributions it is possible to derive $x^{*}$ from (6) explicitly. If $\theta$ is uniformly distributed on $[a, b]$, then it is easy to see that $x^{*}=\frac{C_{1}}{C_{2}} a+\left(1-\frac{C_{1}}{C_{2}}\right) b$ (i.e., $x^{*}$ is between optimistic and pessimistic scenarios of emissions with weights defined by ratio of costs $C_{1}$ and $C_{2}$ ).

\subsection{Comparative analysis}

The Proposition of Section 3.1 allows the comparison of cases of perfect information, full uncertainty, and uncertainty with learning. Equation (6) shows the critical dependence of period 1 optimal decision on the probability distribution $H$. Assume that $C_{1}<C_{2}$. In the case of perfect information, i.e., when $\theta$ is known at the beginning of the first period, both $x_{1}$ and $x_{2}$ can be chosen as a function of observable $\theta$. Clearly, the optimal solution is $x_{1}^{*}=\theta, x_{2}^{*}=0$. Thus, the first term $C_{1} \bar{\theta}$ of the first equation in (7) represents the expected cost under perfect information. The second term represents the expected value of perfect information because this cost would be eliminated if $\theta$ were known before the first-period emission-reduction decision had to be made (rather than afterward as in the learning case). In the case of full uncertainty ("without learning"), the optimal decision $x_{1}=\bar{\theta}, x_{2}=0$ is also known as the certainty equivalent. The possibility of learning combined with explicit introduction of ex post decisions specifies optimal period 1 abatements by the quantile satisfying (6). It may exceed the certainty equivalent $\bar{\theta}$ or it may be below this level. As equation (6) shows, this depends on the relative values of $\operatorname{costs} C_{1}, C_{2}$, and the probability distribution $H$. For example, if $C_{1} / C_{2}=1 / 2$ and $\theta$ has a normal distribution, then optimal ex ante abatement coincides with the certainty equivalent $x_{1}^{*}=\bar{\theta}$. For non-normal probability distributions, the optimal abatements can be below or above $\bar{\theta} .1$

Remark 4: The certainty equivalent solution in the case of full uncertainty (no learning) does not satisfy (4) for all $\theta$, which may lead to a catastrophic collapse of high probability. The only way to fulfill the safety constraint (4) is to choose $x_{1}$ from the

\footnotetext{
${ }^{1}$ An asymmetric probability distribution can be caused, for example, by the interaction of a symmetric probability distribution with an environmental constraint. For example, if the probability density function for $e(\omega)$ is normal, the distribution for $\theta(\omega)$ can still be asymmetric if there is an atmospheric concentration constraint that does not require emissions reductions for all $\omega$.
} 
worst-case scenario as $\max \theta(\omega), \omega \in \Omega$. Clearly, this is an unrealistic and extremely costly solution. This calls for the explicit introduction of safety constraint (2) to provide a trade-off between cost effectiveness and risk. The optimal solution under full uncertainty is now defined as minimal $x_{1}, x_{1}=x_{\gamma}$, satisfying equation $P\left[x_{1} \geq \theta\right]=1-\gamma\left(\right.$ since $\left.C_{1}<C_{2}, x_{2}=0\right)$. Clearly, the risk-based solution under full uncertainty $x_{1}=x_{\gamma}$ may be greater or less than $\bar{\theta}$, depending on $\gamma, C_{1} / C_{2}$, and probability distribution $H$. Imperfect learning (Section 4.2) may shift the ranges (the support) of the probability distribution requiring ex ante abatement that is more or lessaggressive than $x_{\gamma}$.

\section{Uncertain Costs and Outcomes}

\subsection{Uncertain cost}

This section illustrates that the use of expected costs (a common assumption of standard integrated assessment models (Wright and Erickson, 2003)) can be misleading, which calls for the use of quantiles rather than expectations. Assume that stage $2 \operatorname{cost} C_{2}$ in (3) is observable (ex-post) random variable, $C_{2}(\omega), C_{2}=E C_{2}(\omega), C_{1}>0, C_{2}>0$. Function (5) is written as $F(x)=C_{1} x+E C_{2}(\omega) \max \{0, \theta(\omega)-x\}$. The optimal ex ante abatements satisfy equation $F^{\prime}(x)=C_{1}-E C_{2}(\omega) I(\theta \geq x)=0$, i.e., risk attitudes induced among ex ante decisions are now characterized by more complicated risk measures than the CVaR risk measure of Section 3.1. Let us simplify the analysis by assuming as in Arrow and Fisher, (1974) that $\theta=d$, where $d$ is a known (deterministic) positive number. Then, without learning of cost $C_{2}(\omega)$, the problem is to minimize the expected cost $C_{1} x_{1}+C_{2} x_{2}$, where $x_{1}+x_{2}=d, x_{1} \geq 0, x_{2} \geq 0$. If $C_{1}<C_{2}$, then the solution $x_{1}^{*}=d, x_{2}^{*}=0$ is optimal. The ability to learn before the period 2 decision is made implies that a decision maker may reject emissions reduction in period 1 , with a likelihood given by the probability that $C_{2}(\omega)<C_{1}$. This poses the important question about the applicability of expected costs, which is also the key issue for evaluating the potential catastrophic impacts characterized by skewed and multimodal distributions (see, e.g., Figures 5, 7 in Ermoliev et al, 2000). This requires the use of quantiles, say, the median rather then expected values.

Consider random two-period costs $C_{1} x_{1}+C_{2}(\omega)\left(d-x_{1}\right)$. The median of $C_{1} x_{1}+C_{2}(\omega)\left(d-x_{1}\right) \quad$ is $\quad C_{1} x_{1}+\operatorname{med} C_{2}(\omega)\left(d-x_{1}\right)$. Assume that $C_{1}=3.5$, $C_{2}(\omega)=-3$ with probability $1 / 3, C_{2}(\omega)=15$ with probability $1 / 3$, and with probability $1 / 3$ it is uniformly distributed in the interval $[2,4]$. Thus, $E C_{2}(\omega)=5, \operatorname{med} C_{2}(\omega)=3$. Therefore, the optimal solution using expectations without learning is $x_{1}^{*}=d, x_{2}^{*}=0$. Let us notice that the expected value $E C_{2}(\omega)=5$ does not occur in reality, i.e., unlike the median it does not belong to the support of the distribution of $C_{2}(\omega)$. The use of the median leads to the optimal solution $x_{1}^{*}=0, x_{2}^{*}=d$, i.e., it reverses the previous 
conclusion regarding the optimal solution. Assume now that $C_{1}=11, C_{2}(\omega)=-5$ with probability $1 / 5, C_{2}(\omega)=5$ with probability $1 / 5$ and with probability $3 / 5$ it is uniformly distributed in interval $[14,16]$. In this case, $E C_{2}(\omega)=9$, i.e., the optimal solution without learning is $x_{1}^{*}=0, x_{2}^{*}=d$. Since $\operatorname{med} C_{2}(\omega)>14$, then the use of the median leads to the opposite optimal solution $x_{1}^{*}=d, x_{2}^{*}=0$.

Remark 5: An approach for minimizing quantiles follows from Section 3.1. It shows that the minimization of a quantile of $C_{1} x_{1}+C_{2}(\omega) \max \{0, \beta-x\}$ can be reduced to minimization of a convex function $y+\mu E \max \left\{0, C_{1} x+C_{2}(\omega) \max \{0, \beta-x\}-y\right\}$ with respect to $(x, y)$. The solution of this STO problem $\left(x^{*}, y^{*}\right)$ satisfies constraints

$$
P\left[C_{1} x+C_{2}(\omega) \max \{0, \beta-x\} \geq y\right]=\frac{1}{\mu} .
$$

\subsection{Uncertain durations of stages: Limited adaptive capacity}

The general two-stage problem (Section 2) with constraint (2) has a random duration of stages. There are at least two reasons this duration may be uncertain. First, in cases with learning, the timing of new information may be uncertain. For example, if learning is slow, the second period may occur late, while if learning is fast the second period will begin early. In addition, inertia in socio-economic systems may affect the duration of stages. For example, a given emissions reduction in period 1 may take a long time in systems with substantial inertia, but less time in more flexible systems (Ha-Duong et al, 1997). The path-dependencies (inertia) of the socio-economic systems producing greenhouse gasses are critical for dealing with abrupt changes. Without inertia, the switching from one emission path to another would be instantaneous. In reality, energy production systems cannot be changed overnight. As a result, preparedness programs may be only partially implemented because of the potential lack of time in both stages. The proper treatment of these effects requires the explicit introduction of random durations of stages. The following show that a delay with proper ex ante abatements may result in a violation of constraint (2).

In its simplest form, the uncertain duration of stages can be modeled by constraints $x_{2} \leq \beta$ with positive random $\beta$ which becomes known from learning at stage 2 . In other words, it is assumed that the uncertain duration of stage one (with fixed two period time horizon $T$ ) affects the capacity for reductions in period 2 (for example, a longer period 1 reduces the scope for reductions in period 2 given its shorter duration).

Without the safety constraint of type (2), the optimal stage 2 decision $x_{2}=\min \left[\beta, \max \left\{0, \theta-x_{1}\right\}\right]$ cannot in general satisfy safety constraints (4) for all $\theta$. As a consequence, the probability of a catastrophe can be rather high, calling for explicit introduction of type (2) safety constraint $P\left[x_{1}+x_{2} \geq \theta\right]=1-\gamma$. Since $x_{2} \leq \beta$, this requires ex-ante emission reduction commitments $x_{1} \geq x_{\gamma}$, where $x_{\gamma}$ is minimal nonnegative $x$ satisfying equation $P[x \geq \theta-\beta]=1-\gamma$. Therefore, in order to prevent a 
catastrophic collapse, there must exist regulations on minimal ex-ante emission reductions sufficient to keep open the possibility of satisfying the safety constraint in stage 2 , which can be evaluated by analyzing STO problems with safety constraints (2).

\subsection{Incomplete learning and safety constraints}

Consider a very realistic case where the learning affects only the prior distribution $H(z)$, in other words, it shifts the range of uncertainty. As the optimal period 1 decision of Section 3.1 is a quantile of $H(z)$, learning may dramatically affect this decision in different directions. Let us assume that $H(z)=P[\theta \leq z]$ is a mixture $H(z)=E_{\xi} H(\xi, z)=\int H(y, z) d G(y)$ of distribution $H(\xi, z)$ with unknown $\xi$ characterized by a probability distribution $G(y)=P[\xi \leq y]$, which may reflect polarized views on scenarios of climate changes. The learning reveals only $\xi$ at the beginning of period 2. For example, $H(z)$ can be a mixture of distributions $H(\xi, z)$ with probability mass concentrated in different subregions from the support of $H(z)$. If the support of $H(\xi, z)$ is a singleton, then the learning of $\xi$ reveals the true value of $\theta$. For the sake of illustration, let $H(z)$ be a mixture of two distributions $H_{0}(z)$ and $H_{1}(z)$, that is, $\xi H_{0}(z) \dashv(1-\xi) H_{1}(z)$, where $\xi=0$ with probability $p$ and $\xi=1$ with probability $1-p$, that is, $H(z)=p H_{0}(z)+(1-p) H_{1}(z)$. Since only $\xi$ is observed, the stage 2 decision $x_{2}(\xi)$ can not fulfill constraints (4), and the safety constraint has to be written as in (2):

$$
P\left[x_{1}+x_{2}(\xi) \geq \theta(\xi)\right] \geq 1-\gamma,
$$

where $\theta(\xi)$ has the probability distribution $H_{\xi}(z)$. For a given $\xi$ and $\gamma$, let us define $z_{\gamma}(\xi)$ as minimal $z$, satisfying equation $P[z \geq \theta(\xi)]=1-\gamma$. Then equation (8) is equivalent to the equation $x_{1}+x_{2}(\xi) \geq z_{\gamma}(\xi)$, which is similar to (4). The optimal period 2 decision $x_{2}=\max \left\{0, z_{\gamma}(\xi)-x_{1}\right\}$, and optimal $x_{1}$ has to minimize $F(x)=C_{1} x+C_{2}\left[p \max \left\{0, z_{\gamma}(0)-x\right\}+(1-p) \max \left\{0, z_{\gamma}(1)-x\right\}\right]$.

As $\xi$ has a discrete probability distribution, function $F(x)$ does not have continuous derivatives. Therefore, the optimality condition cannot be derived from the Proposition of Section 3.1. Assume that $z_{\gamma}(0)<z_{\gamma}(1) . F(x)$ is a piece-wise continuous linear function. Namely, for $0 \leq x<z_{\gamma}(0)$,

$$
F(x)=C_{1} x+C_{2}\left[p\left(z_{\gamma}(0)-x\right)+(1-p)\left(z_{\gamma}(1)-x\right)\right]=\left(C_{1}-C_{2}\right) x+C_{2} \bar{z}_{\gamma}(\xi) .
$$

For $z_{\gamma}(0) \leq x<z_{\gamma}(1)$, 


$$
F(x)=C_{1} x+C_{2}(1-p)\left(z_{\gamma}(1)-x\right)=\left(C_{1}-C_{2}(1-p)\right) x+C_{2}(1-p) z_{\gamma}(1)
$$

and

for

$x \geq z_{\gamma}(1), F(x)=C_{1} x$.

The optimal ex-ante solution hedges against different contingencies. It is characterized as follows: $x=0$, if $C_{1}>C_{2}$. Otherwise, $x=z_{\gamma}(0)$, if $C_{1}-C_{2}(1-p)>0$, and $x=z_{\gamma}(1)$, if $C_{1}-C_{2}(1-p)<0$.

Since $C_{1}<C_{2}$, in general it pays to make reductions in period 1 that are as large as possible. After learning takes place at the end of period 1, the optimal solution is to make reductions such that the total reduction is either $z(0)$ or $z(1)$. Thus, the minimal first period reduction is $z(0)$. If first period costs are very low, or the chance that $\xi=1$ is very high, then it is optimal to make larger first period reduction $z(1)$, accepting the chance that $\xi=0$ and that reductions $z(1)-z(0)$ will have been unnecessary.

Let us compare this ex ante period 1 optimal "with-learning" solution with the optimal "without-learning" solution $x_{1}^{*}=z_{\gamma}, x_{2}^{*}=0$ derived from minimization of (4) under safety constraint $P\left[x_{1}+x_{2} \geq \theta\right] \geq 1-\gamma$, i.e., $x_{1}+x_{2} \geq z_{\gamma}$, where $z_{\gamma}$ is the minimal $z$ satisfying constraint $P[z \geq \theta] \geq 1-\gamma$. Assume that $H_{0}(z), H_{1}(z)$ have continuous derivatives, the support of distribution $H_{0}(z)$ is interval $\left[a_{0}, b_{0}\right]$, and the support of $H_{1}(z)$ is interval $\left[a_{1}, b_{1}\right]$, where $a_{1}>b_{0}$. If $C_{1}-C_{2}(1-p)<0$, then the optimal "with-learning" period 1 solution $x=z_{\gamma}(1)$. If $x \in\left[a_{1}, b_{1}\right]$, then $P[x \geq \theta]=p+(1-p) H_{1}(x) . \quad$ Since $\quad H_{1}\left(z_{\gamma}(1)\right)=1-\gamma, \quad$ then $P[x \geq \theta]=p+(1-p)(1-\gamma)=1-\gamma+\gamma p$ for $x=z_{\gamma}(1)$. As $\gamma p>0$, then the optimal "without-learning" decision $x=\theta_{\gamma} \quad$ satisfying $P[x \geq \theta]=1-\gamma$ is less demanding (smaller) than $x=z_{\gamma}(1)$, i.e., learning increases the optimal ex ante emission reductions. This conclusion is reversed in the case $C_{1}-C_{2}(1-p)>0$. Indeed, let $x \in\left[a_{0}, b_{0}\right]$. Then, $P[x \geq \theta]=p H_{0}(x), \quad H_{0}\left(z_{\gamma}(0)\right)=1-\gamma \quad$ and for $\quad x=z_{\gamma}(0)$, $P[x \geq \theta]=p(1-\gamma)$ (i.e., the optimal "without-learning" decision $x=\theta_{\gamma}$ is greater than the optimal "with-learning" decision $\left.x=z_{\gamma}(0)\right)$.

\section{Nonlinear Abatement Cost}

It is well known that abatement-cost functions are nonlinear (Ha-Duong et al, 1997). This section illustrates that, in contrast to the linear case, nonlinear cost functions call more strongly for the coexistence of ex ante and ex post decisions. It is interesting to compare the case of linear functions with the quadratic cost functions used in some integrated assessment models. Assume that the cost functions of both periods $C_{i}(x)=C_{i} x^{2}$ with positive $C_{1}, C_{2}$. Cost function (4) takes on the form 
$F(x)=C_{1} x^{2}+C_{2} E(\max \{0, \theta-x\})^{2} \quad$ and $\quad F^{\prime}(x)=2 C_{1} x-2 C_{2} E(\theta-x) I[\theta \geq x]$.

Therefore, $F^{\prime}(0)=-2 C_{2} E \theta<0$, assuming that $E \theta>0$ with a positive probability. Thus, this case calls for coexistence of period 1 and period 2 decisions independently of $C_{1}, C_{2}$. Compare this to the case of linear costs in Section 3.1, where $F^{\prime}(0)=C_{1}-C_{2}$ if $C_{1}<C_{2}$; i.e., non-zero first period reductions are called for only if costs are less in period 1. With quadratic costs, period 1 reductions are optimal even if $C_{1}>C_{2}$ because marginal costs can still be lower in period 1 if larger period 2 reductions will be required.

Optimal ex ante abatements are characterized by more complicated than (6) equation $C_{1} x=C_{2} E(\theta-x) I(\theta \geq x)$. In the case of normally distributed $\theta$, $F(x)=C_{1} x^{2}+\frac{1}{2} C_{2} E(\theta-x)^{2}$. From the optimality $2 C_{1} x+C_{2}(x-E \theta)=0$ it follows that optimal $x=C_{2} \bar{\theta} /\left(2 C_{1}+C_{2}\right)$, i.e., it is defined only by mean value $\bar{\theta}$. This conclusion is false for non-normal distributions, although standard integrated assessment models often use only average values $\bar{\theta}$.

It is important to illustrate some typical situations that may occur in the case of non-smooth, piece-wise linear functions commonly used in emission-control problems where technology switches may call for more intensive emission reduction. These functions implicitly impose upper or lower bounds on ex ante emission reductions. Assume that $C_{2}(x)=C_{2} x$ and $C_{1}(x)$ is a piece-wise linear function $C_{1}(x)=C_{1}^{1} x$ for $0 \leq x \leq a$ and $C_{1}(x)=C_{1}^{2}(x-a)+C_{1}^{1} a$ for $x \geq a$, where $C_{1}^{2}>C_{2}$ and $C_{1}^{1}<C_{2}$. It is easy to see that the optimal ex ante solution has to satisfy the additional requirement $x_{1} \leq a$. As $C_{1}^{1}<C_{2}$ and $C_{1}^{2}>C_{2}$, the optimal ex ante decision is defined as follows: let $\bar{x}$ be the solution of equation $P[\theta>x]=C_{1}^{1} / C_{2}$. The optimal period 1 decision $x_{1}^{*}=a$ if $\bar{x}>a$, and $x_{1}^{*}=\bar{x}$ for $\bar{x} \leq a$. Assume that $C_{1}(x)=C_{1} x$, and $C_{2}(x)=C_{2}^{1} x$ for $0 \leq x \leq a ; C_{2}(x)=C_{2}^{2}(x-a)+C_{2}^{1} a$ for $x \geq a$, where $C_{1}>C_{2}^{1}, C_{1}<C_{2}^{2}$. Consider solution $\underline{x}$ of the equation $P[\theta>x]=C_{1}^{1} / C_{2}$. It is easy to see that the optimal period-1 decision $x_{1}^{*}=\underline{x}$ for $\underline{x} \geq a$ and $x_{1}^{*}=0$ for $\underline{x}<a$.

\section{A Dynamic Stabilization Problem}

The general stabilization problem is outlined in Section 2. Its proper formulation requires a catastrophe-generating submodel (i.e., a submodel of $\mathrm{CO}_{2}$-generating activities). Therefore, in its rather general form, the problem becomes similar to catastrophic-risk-management problems discussed in [9]. In its simplest form, the dynamic two-stage model has strong connections with dynamic versions of CVaR risk measures. 
Assume that $\mathrm{CO}_{2}$ emission paths are characterized by exogenous scenarios as in Section 3. Let us consider $R_{t}=\sum_{k=0}^{t} x_{k}$, where decision variables $x_{k} \geq 0, k=0,1, . ., t$, $t \leq T$. We can think of $x_{k}$ as a feasible level of $\mathrm{CO}_{2}$ emission reduction at the beginning of period $k$. At time $t=0$ the target value on total emission reduction $R_{t}$ in period $t$ is given as a random variable $\rho_{t}$. It is assumed that the exact value of $\rho_{t}$ is revealed at a random time $t=\tau$. Since $\tau$ is uncertain, the decision path $x=\left(x_{0}, x_{1}, \ldots, x_{T}\right)$ for the whole time horizon has to be chosen ex ante in period $t=0$ to "hit" the target $\rho_{t}, R_{\tau} \geq \rho_{\tau}$, at $t=\tau$ in a sense specified further by (10). At random $t=\tau$, the decision path can be revised for the remaining available time. Similar to the model of Section 3.1, consider a stream of linear random costs $v(x)=\sum_{t=0}^{T}\left[c_{t} x_{t}+d_{t} \max \left\{0, \rho_{t}-R_{t}\right\}\right] I_{\tau=t}$, where $c_{t}>0, \quad d_{t}>0, \quad t=0,1, \ldots, T \quad$ are known ex-ante and ex-post abatement costs. The expected value of $v(x)$ can be written as

$$
V(x)=\sum_{t=0}^{T} c_{t} x_{t}+E d_{\tau} \max \left\{0, \rho_{\tau}-\sum_{t=0}^{\tau} x_{t}\right\}
$$

Let us consider a path $x^{*}$ minimizing $V(x)$ subject to $x_{t} \geq 0, t=0,1, \ldots, T$. Assume that $V(x)$ is a continuously differentiable function (e.g., a component of random vector $\rho=\left(\rho_{0}, \rho_{1}, \ldots, \rho_{T}\right)$ has a continuous density function). Assume also that, so far, there exists a positive optimal solution $x^{*}=\left(x_{0}^{*}, x_{1}^{*}, \ldots, x_{T}^{*}\right), x_{t}^{*}>0, t=0,1, \ldots, T$. Then, from the optimality condition for stochastic minimax problems (see, e.g., discussion in [4], [10]), it follows that for $x=x^{*}$, $V_{x_{t}}=c_{t}-\sum_{k=0}^{t} p_{k} d_{k} E I\left(\sum_{k=0}^{t} x_{k} \leq \rho_{k}\right)$ or $V_{x_{t}}=c_{t}-\sum_{k=0}^{t} p_{k} d_{k} \mathrm{P}\left[\sum_{k=0}^{t} x_{k} \leq \rho_{k}\right]=0, t=0,1, \ldots, T$, where $p_{t}$ is the probability that $\tau$ occurs first time at $t$. From this sequentially for $t=0,1, \ldots, T$, it follows that

$$
P\left[x_{0}<\rho_{0}\right]=c_{0} / p_{0} d_{0}, P\left[\sum_{k=0}^{t} x_{k}<\rho_{k}\right]=\left(c_{t}-c_{t-1}\right) / p_{t} d_{t}, t=0,1, \ldots, T .
$$

It is easy to see that from (9) it follows that

$$
V\left(x^{*}\right)=c_{0} E\left[\rho_{0} I\left(\rho_{0}>R_{0}^{*}\right)\right]+\left(c_{1}-c_{0}\right) E\left[\rho_{i} I\left(\rho_{1}>R_{1}^{*}\right)\right]+\ldots+\left(c_{T}-c_{T-1}\right) E\left[\rho_{T} I\left(\rho_{T}>R_{T}^{*}\right)\right\rfloor
$$
which can be viewed as a dynamic $\mathrm{CVaR}$ risk measure.

Remark 6: Equations (9) are derived from the existence of the positive optimal solution $x^{*}$. It is easy to see that this solution follows from $c_{0} / p_{0} d_{0}<1$, $\left(c_{t}-c_{t-1}\right) / p_{t} d_{t}<1$ and the monotonicity of quantiles $\beta_{t}, \beta_{0}<\beta_{1}<\ldots<\beta_{T}$ defined by equations

$$
P\left[\beta_{0}<\rho_{0}\right]=c_{0} / p_{0} d_{0}, P\left[\beta_{t}<\rho_{t}\right]=\left(c_{t}-c_{t-1}\right) / p_{t} d_{t}, t=0,1, \ldots, T .
$$


If probability $p_{t}$ rapidly decreases to 0 , then from (9) it follows that ex-ante abatements are positive for a relatively short initial interval defined by inequality $\left(c_{t}-c_{t-1}\right) / p_{t} d_{t}<1$. This misleading conclusion is due to a strong assumption of unlimited capacity for emissions reductions, which is a standard assumption of climaticeconomic integrated assessment models (see discussion in Ha-Duong, 1997 and Wright and Erickson, 2003). Similar to conclusions of Section 4.2, this requires an adequate treatment of safety constraints (2) to prevent catastrophes.

\section{Concluding Remarks}

This paper analyzes the effects of risks and learning on climate change decisions using a two-stage, dynamic model that assumes a stabilization constraint. It shows that learning can lead either to larger or smaller first period emissions reductions, compared to the optimal reduction under uncertainty without learning, and that this effect can either be large or small. The direction and magnitude of the learning effect is determined by a number of interacting factors. For example, in a simple linear model with deterministic mitigation costs but uncertainty in total required emissions reductions, the learning effect depends on how mitigation costs evolve over time, the shape of the uncertainty distribution in required emissions reductions, the confidence with which the safety constraint (i.e., stabilization level) is desired to be met, and, in the case of incomplete learning, the probability distribution describing the anticipated learning possibilities. If costs are uncertain, but the emissions reduction target is known, the problem emphasizes the use of risk adjusted costs (e.g., quantiles) rather than expected values. Introducing a more realistic nonlinear cost function with increasing marginal costs induces a higher level of first period emissions reductions compared to the linear case. We also analyze the case of random duration of stages as a proxy either for uncertain timing of learning or uncertain inertia in socio-economic systems, showing how this consideration can induce a minimum level of first period reductions. Finally, framing the problem in dynamic terms as a multi-period problem with an uncertain time path of required cumulative emissions reductions shows that the problem has strong connections with dynamic versions of $\mathrm{VaR}$ risk measures. Given the multiple influences on the learning effect, we conclude that drawing practical conclusions on the likely effect of learning on climate change decisions is an empirical question requiring analysis with models capable of adequately representing endogenous risks, abrupt changes, in particular, abrupt learning, inertia, and path dependent costs.

\section{References}

Arrow, K.J. and A. Fisher. 1974. Environmental preservation, uncertainty, and irreversibility. Quarterly Journal of Economics 88: 312-319.

Chichilnisky, G. and G. Heal. 1993. Global environmental risks. Journal of Economic Perspectives 7(4): 65-86.

Clarke, F.H. 1983. Optimization and Nonsmooth Analysis. Wiley, New York.

Dantzig, G. and A. Madansky. 1961. On the solution of two-stage linear programs under uncertainty. Proc. Fourth Berkeley Symposium on Mathematical Statistics and Probability. Volume 1, University California Press, Berkeley, pages 165-176. 
Dixit, A.K. and R.S. Pindyck. 1994. Investments under uncertainty. Princeton University Press.

Embrechts, P., C. Klueppelberg, and T. Mikosch. 2000. Modeling Extremal Events for Insurance and Finance. Applications of Mathematics, Stochastic Modeling and Applied Probability, Springer Verlag, Heidelberg.

Epstein, L.G. 1980. Decision making and the temporal resolution of uncertainty. International Economic Review 21(2): 269-282.

Ermoliev, Y., T.Y. Ermolieva, G.MacDonald, and V.Norkin. 2000. Stochastic optimization of insurance portfolios for managing exposure to catastrophic risks. Annals of Operations Research 99: 207-225.

Ermoliev, Y.M. and V.I. Norkin. 1997. On nonsmooth and discontinuous problems of stochastic systems optimization. European Journal of European Research 101: 230-244.

Ermoliev, Y. and R.Wets (eds.) 1988. Numerical Techniques for Stochastic Optimization. Computational Mathematics, Springer Verlag, Berlin.

Fisher, A.C. and U. Narain. 2003. Global warming, endogenous risk, and irreversibility. Environmental and Resource Economics 25: 395-416. Kluwer, Netherlands.

Ha-Duong, M., J-C. Hourcade, and M.Grubb. 1997. The influence of inertia and uncertainty upon optimal $\mathrm{CO}_{2}$ policies. Nature 390: 270-274.

Henry, C. 1974. Investment decisions under uncertainty: the irreversibility effect. American Economic Review 64(6): 1006-12.

Kall, P. and S.W. Wallace. 1994. Stochastic Programming. J. Wiley, Chichester.

Kolstad, C.D. 1996. Learning and stock effects in environmental regulations: the case of greenhouse gas emissions. Journal of Environmental Economics and Management 31:1-18.

Manne, A.S. and R.G. Richels. 1992. Buying Greenhouse Insurance: The Economic Costs of Carbon Dioxide Emission Limits. . MIT Press, Cambridge MA.

Marti, K. 2005. Stochastic Optimization Methods. Springer, Berlin, Heidelberg.

Nordhaus, W.D. 1994. Managing the Global Commons: The Economics of Climate Change. MIT Press, Cambridge MA.

O'Neill, B.C. and M. Oppenheimer. 2002. Dangerous climate impacts and the Kyoto Protocol. Science 296: 1971-1972.

Pindyck, R.S. 1999. Irreversibilities and the Timing of Environmental Policy. Working Paper 99-005. Center for Energy and Environmental Policy Research, Massachusetts Institute of Technology, Cambridge MA.

Rockafellar, T. and S. Uryasev. 2000. Optimization of conditional value-at-risk. The Journal of Risk 2(3): 21-41.

Schultz, P.A. and J.F. Kasting. 1997. Optimal reduction in $\mathrm{CO}_{2}$ emissions. Energy Policy 25(5): 491-500. 
Ulph, A. and D. Ulph. 1997. Global warming, irreversibility and learning. Economic Journal 107(442): 636-650.

Viscusi, W.K. and R. Zeckhauser. 1976. Environmental policy choice under uncertainty. Journal of Environmental and Economic Management 3: 97-112.

Webster, M. 2002. The curious role of "learning" in climate policy: should we wait for more data? The Energy Journal 23(2): 97-119.

Wright, E.L. and J.D. Erickson. 2003. Incorporating catastrophes into integrated assessment: science, impacts, and adaptation. Climate Change 57: 265-286.

Yastremskij, A. 1983. Stochastic Models of Mathematical Economics. Vischa Shkola, Kiev (In Russian). 